\title{
Surfactant adsorption at the Metal-Oil Interface
}

\author{
Mario Campana ${ }^{1}$, Anke Teichert ${ }^{2,5,6}$, Stuart Clarke ${ }^{3}$, Roland Steitz ${ }^{2}$,
} John. R. P. Webster ${ }^{4}$ and Ali Zarbakhsh ${ }^{1 *}$

${ }^{1}$ School of Biological \& Chemical Sciences, Queen Mary, University of London, Joseph Priestley Building, Mile End Road, London E1 4NS, United Kingdom.

${ }^{2}$ Helmholtz-Zentrum Berlin für Materialien und Energie GmbH, Hahn-Meitner-Platz 1, D-14109 Berlin, Germany.

${ }^{3}$ BP Institute, Bullard Laboratories, Madingley Road, Cambridge, CB3 0EZ, United Kingdom.

${ }^{4}$ ISIS Neutron Facility, Science and Technology Facilities Council, Rutherford Appleton Laboratory, Harwell Science and Innovation Campus, Didcot, OX11 0QX, United Kingdom.

${ }^{5}$ Instituut voor Kern- en Stralingsfysica and INPAC, K.U.Leuven, Celestijnenlaan 200D, B-3001 Leuven, Belgium.

${ }^{6}$ Laboratorium voor Vaste-Stoffysica en Magnetisme and INPAC, K.U.Leuven, Celestijnenlaan 200D, B-3001 Leuven, Belgium.

*Author to whom correspondence should be addressed. Email: a.zarbakhsh@qmul.ac.uk 


\begin{abstract}
The structure of the adsorbed palmitic acid at the Fe oxide/oil interface has been investigated using polarised neutron reflectometry. The palmitic acid was found to be strongly adsorbed at the oxide/oil interface resulting in a monolayer of thickness $16 \pm 4 \AA$ for 150 , and 500 ppm palmitic acid concentrations ( $16 \pm 5 \AA$ for the $1000 \mathrm{ppm}$ solution). These layer thicknesses suggest tilt for the palmitic acid molecules with respect to the interface. The model also requires a second diffuse layer extending in the bulk oil. The thickness of this diffuse layer was $35 \pm 17 \AA$ for the $150 \mathrm{ppm}$ and $45 \pm 22 \AA$ for 500 and $1000 \mathrm{ppm}$ solutions. The composition profiles at the interface suggest a depletion of the oil in the vicinity of the interface as the concentration of palmitic acid increases.
\end{abstract}

\title{
Introduction
}

The lubrication of surfaces by oils is a key aspect of many academic and industrial problems with significant financial implications [1]. Although lubrication has been exploited for many centuries, the details of the underlying physics and chemistry are still to be resolved. There are three broad regimes of friction/lubrication depending upon the applied load [2]: from (i) the hydrodynamic regime, where a reasonable layer of oil can survive between the moving surfaces to (ii) the boundary regime where the high load pushes most oil out leaving a molecularly thin film between the surfaces and (iii) an intermediate 'mixed' regime. In many commercial applications, additives (boundary lubricants) can be added to the oil to maintain and enhance the oil monolayer adsorbed on surfaces, which are believed to act by adsorption to the metal surfaces. The physisorption or chemisorptions of long chain polar organic molecules on metal surfaces are considered to form a thin film between the moving metal surfaces which can result in a reduction in the coefficient of friction. A simple example of these additives could be fatty acids adsorbed from non-polar hydrocarbon oils onto a metal surface. Other additives are also included in these systems for example to prevent oxidation and dispersal of particulates. In many cases these are also interfacially active agents.

This area of science is growing in importance because of: Advances in engine technology combined with lower emission requirements; the demand for fuel efficiency and improved friction reduction; and the desire for a longer oil change interval. Hence, understanding the role and the adsorption isotherms for lubricant additives at the oil-metal interface is essential. The measurements outlined below will help towards achieving these goals to improve design and establish a more efficient formulation. 
The adsorption of surfactants at solid-aqueous and air-aqueous solutions has been studied indepth over the past decade using techniques such as X-ray and neutron reflectometry, ellipsometry and surface tension measurements [3-5]. Although surfactants are widely used as friction modifiers, very little work has been done in structural studies of surfactants at nonaqueous metal interfaces. These systems are interesting both in terms of fundamental adsorption processes as well as technological applications. Friction modifiers are normally alkyl species with 10 or more carbons and interfacially active head groups. In addition other agents such as anti-wear additives [6,7] are also added to lubricant fluid (essentially mineral oils, esters and related base fluids). These are typified by zinc dialkyl dithiophosphate (ZDDP). The decomposition of the anti-wear modifiers and their adsorption in the presence of surfactant-friction modifiers are complex and are believed to consist of a complex multilayer structure which is again not fully understood.

In this study we use polarised neutron reflectometry to study the palmitic acid $\left(\mathrm{CH}_{3}\left(\mathrm{CH}_{2}\right)_{14} \mathrm{COOH}\right)$ adsorption at the $\mathrm{Fe}$ oxide/oil interface for a series of surfactant concentrations. This study is an initial part of a larger investigation into understanding the role of adsorbed surfactants and anti-wear inhibitors used in industry.

\section{Materials}

Hexadecane-d34 was obtained from Cambridge Isotope Laboratories (> 98 atom D\%). Palmitic acid $\left(\mathrm{CH}_{3}\left(\mathrm{CH}_{2}\right)_{14} \mathrm{COOH}\right)$ was purchased from Sigma-Aldrich UK. The substrates kept in an inert atmosphere prior to the neutron experiments. All solutions and mixtures were prepared by mass. An iron layer was deposited on a silicon substrate $(55 \mathrm{~mm}$ diameter) together with a reference Fe layer coated float glass ( $3 \mathrm{~mm}$ thick) substrate for the purpose of calibration of the sputtering machine. All the sample depositions were carried out at Helmholtz Zentrum Berlin using a UHV DC magnetron sputtering system with the argon pressure and sputter power of $1.3 \times 10^{-3}$ mbar and $0.65 \mathrm{~kW}$ respectively (the base pressure was $\left.6.5 \times 10^{-6} \mathrm{mbar}\right)$.

\section{Experimental}

A non-magnetic, small volume cell $(\sim 1 \mathrm{ml})$ was constructed from aluminium with plastic screws for the polarised neutron reflectometry experiment. The cell is shown schematically in Figure 1. Polarised neutron reflectometry was solely used as a means of providing additional contrasts. The polarised neutron reflectometry experiment was 
undertaken using the neutron reflectometer, V6, at the Helmholtz Zentrum Berlin, Germany. The V6 instrument deploys a cold neutron wavelength of $4.66 \AA$ together with a polarisation ratio of $98.5 \%$. The instrument uses pyrolytic graphite crystals as a monochromator together with a liquid nitrogen cooled Be filter positioned before the sample. The polarised monochromatic neutrons are produced using a Si-FeCo supermirror in the incident beam. The beam collimation is achieved by using two sets of computer controlled cadmium slits. A magnetic field in the sample plane is applied to the sample perpendicular to the neutron beam direction. The neutron detector used was an array of ${ }^{3} \mathrm{He}$ gas detectors moving in a vertical plane.

Neutron reflectivity is a technique sensitive to the average neutron refractive index, $n$, profile normal to an interface. The neutron refractive index for a magnetic sample which is magnetised in the plane of the surface can be written as a sum of the nuclear $\left(n_{\text {nuclear }}\right)$ contribution and the magnetic $\left(n_{\text {magnetic }}\right)$ component of the sample. The refractive index for a magnetised sample in a neutron beam polarised parallel $(\delta+)$ and antiparallel $(\delta-)$ to a field applied to the sample can be written as [8]

$$
\begin{gathered}
n_{ \pm}(\lambda)=n_{\text {nuclear }}(\lambda) \pm n_{\text {magnetic }}(\lambda) \\
n_{ \pm}(\lambda)=\left[1-\frac{N \lambda^{2}(b \pm C \mu)}{2 \pi}\right]
\end{gathered}
$$

where $\lambda$ is the neutron wavelength, with $N$ the number density, $b$ the coherent nuclear scattering length, and $\mu$ is the average magnetic moment per atom and $\mathrm{C}$ is a constant $(0.2645$

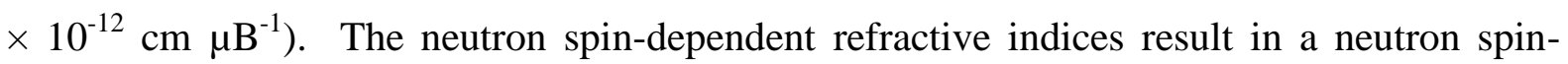
dependent reflectivity $\left(\mathrm{R}^{+}\right.$and $\left.\mathrm{R}^{-}\right)$. The reflectivities can be calculated using, among other methods, the standard optical-matrix method and the parameters of the proposed layer model can be optimised using non-linear least-squares fitting.

\section{Results}

The X-ray reflectivity (the wavelength and resolution $1.54 \AA$ and $0.004 \AA^{-1}$ respectively) from the calibration iron sample on a float glass is shown in Figure 2. The fit to the data is shown by the solid line and the fitted parameters are given in Table 1. As usual in these experiments, to provide adhesion of the sputtered iron layer on the glass substrate an addition layer of tin $(10 \pm 4 \AA)$ was used. This layer was only required for the float glass reference and not for the Silicon. Subsequently a Si-Fe sample with a layer of $30 \mathrm{~nm}$ was 
investigated with polarised neutron reflectometry. The bare iron surface of the Si-Fe system was first characterised at the air/iron interface prior to the surfactant adsorption experiment at the soild-liquid interface. The neutron spin flip asymmetery for the iron sample at the metal/air interface is shown in Figure 3. The solid line shows the fit corresponding to Fe layer of $302 \pm 5 \AA$ layer with roughness of $6 \AA$ and an iron oxide layer of $28 \pm 2 \AA$ and roughness of $6 \AA$. The insert Figure (Fig 3) shows the individual spin up $(\delta+)$ and spin down $(\delta-)$ reflectivity profiles. The fits to data using the model just described are shown by the solid line and the fitted parameters are given in Table 2, and are in very good agreement with the experimental data. The scattering length density of the oxide layer suggests it consist of a mixture of oxides, namely $\mathrm{Fe}_{2} \mathrm{O}_{3}$ (non-magnetic) and $\mathrm{Fe}_{3} \mathrm{O}_{4}$ (magnetic). Mixtures of oxides have been reported for the sputtered iron layers in literature [9]. The fitted parameters for the substrate including the oxide layer are kept constant for all subsequent fittings and the relative changes in the reflectivity profiles as the function of palmitic acid concentrations are not affected.

The iron coated Si substrate was then mounted on the sample cell and filled with oil. A series of polarised neutron reflectivity profiles were measured as a function of palmitic acid concentration in deuterated hexadecane at room temperature. A magnetic field of 300 Gauss ( 0.03 Tesla) was applied in the plane of the sample perpendicular to the direction of the incident neutron beam. The schematic diagram of the reflection geometry used is shown in Figure 4(a). The idealised scattering length density profiles for both the magnetic and the nuclear contributions of $\mathrm{Si}$, iron and iron oxide layers with hexadecane forming the bulk oil are shown in Figure 4(b). These scattering length density profiles clearly show that for the spin up $(\delta+)$ neutrons the reflectivity would strongly be dominated by the $\mathrm{Si} /$ iron interface contribution, hence a reduction in sensitivity to any adsorbed palmitic acid at the iron oxide/ bulk oil interface. Hence it is the spin down $(\delta$-) reflectivites that will provide the optimum contrast scheme for resolving the structure of the palmitic acid at the iron oxide/bulk oil interface.

The spin up $(\delta+)$ and spin down $(\delta-)$ reflectvities $\left(\mathrm{R}^{+}\right.$and $\left.\mathrm{R}^{-}\right)$were measured for all concentrations and an example of a pair is shown for $150 \mathrm{ppm}$ palmitic acid concentration in Figure 5. Henceforth only the spin down $(\delta$-) reflectivity profiles are shown for convenience. The parameters deduced for the bare substrate were fixed in all subsequent fitting procedures. The reflectivity profiles for spin down neutrons $(\delta-)$ for a series of palmitic acid concentrations (150 to $1000 \mathrm{ppm}$ ) are shown in Figure 6 with d-hexadecane forming the bulk 
oil solution. The fits to these data are shown by the solid lines. The fitted parameters for these fits are given in Table 3.

The scattering length density representation of the fitted models is shown in Figure 7(a). The volume fraction profiles for the oil (hexadecane) and the corresponding palmitic acid as a function of distance away from the iron oxide are shown in Figure 7(b) and 7(b) insert respectively.

\section{Discussion}

The fitted parameters to the reflectivity profiles for all the palmitic acid concentrations suggested some degree of porosity for the iron oxide layer. The scattering length density of the iron oxide layer indicated some inclusion of the palmitic acid to a depth of $8 \pm 2 \AA$ (the overall thickness of the oxide layer was $28 \pm 6 \AA$ ). The overall composition of the oxide layer was the same for all the palmitic acid concentrations. A two layer model was then required to fully describe the extent and the structure of the adsorbed palmitic acid at the iron oxide/oil interface. The palmitic acid was found to be strongly adsorbed on the oxide/oil interface resulting in a monolayer of thickness $16 \pm 4 \AA$ for 150 , and 500 ppm palmitic acid concentrations (16 $\pm 5 \AA$ for the 1000 ppm solution). The fully extended molecular length for this acid is $\sim 21 \AA$ layer, hence these layer thicknesses suggest an apparent tilt of the palmitic acid molecules with respect to the interface normal. The model also requires a second diffuse layer extending in the bulk oil. The thickness of this diffuse layer was $35 \pm 17 \AA$ for the 150 ppm and $45 \pm 22 \AA$ for 500 and 1000 ppm solutions. The scattering length density and composition of these layers are shown in Figure 7(a). The composition profiles, Figure 7(b), suggest a depletion of the oil in the vicinity of the interface as the concentration of palmitic acid increases. The fitted parameters are tabulated in Table 3.

The roughness of the second diffuse layer is relatively high, however acceptable $(<1 / 2$ the layer thickness). This structure represents a gradual change in composition of the palmitic acid away from the interface as indicated by the composition profiles, Figure 7(b) (insert). The adsorbed amounts were calculated by integrating the volume fraction profiles of the palmitic acids and are shown in Figure 8(a). This data shows an almost linear increase for the palmitic acid adsorbed amount as a function of concentration used.

The wear profile (reported in the literature [10]) for the palmitic acid in n-hexadecane is shown in Figure 8(b). There is a good correlation between this profile and adsorbed amount determined in this report. The profile suggests that as the adsorbed amount of the palmitic 
acid increases with concentration, it results in the exclusion of the oil in the vicinity of the metal oxide surface. This exclusion is detrimental to the hydrodynamic lubrication process provided by the oil. The role of fatty acid in these systems has been suggested to arise from increase in wettability of the oil at the metal surface. At a low adsorbed amount of palmitic acid the metal oxide oil wettability increases providing the condition of good lubrication. As the adsorbed amount of acid increases prior to a full coverage there is depletion of the oil in the vicinity of the metal oxide/bulk oil interface and this is reflected in the increase of wear observed in the relative wear profile. However when a full coverage is reached the lubrication property recovers. This indicates a fully hydrophobic surface is now formed by the acid allowing a complete wetting of the surface by the hexadecane oil, hence a recovery in the lubrication process and improved wear as suggested by the wear profile of Figure 8(b).

\section{Conclusion}

This paper reports to our best of our knowledge, the first measurements of the adsorption of surfactants at the metal oxide/oil interface as a function of surfactant concentration using polarised neutron reflectometry. We have used polarised neutrons in order to provide important additional contrast that is key in achieving structural solution in such complex systems. We found that the adsorbed amount for palmitic acid increases almost linearly with the concentration.

It is possible to consider the relevance of such adsorption in industrial problems such as lubrication and wear, and we have outlined some tentative suggestions that may connect the adsorption behaviour and wear of this fatty acid.

In our future experiments we will extend this study to investigate other surfactants and oil systems, particularly multicomponent mixtures, in addition to assessing the role of temperature on these adsorption profiles.

\section{Acknowledgements}

This project has been supported by the European Commission under the seventh Framework Programme through "Research Infrastructures" action of the "Capacities" Programme, contract number CP-CSA_INFRA-2008-1.1.1. Number 226507-NMI3. We thank the Helmholtz Zentrum Berlin, Germany for allocation of beam time and access to support and facilities and our thanks to Dr Timothy Charlton and our colleagues at BP Castrol for helpful discussions. 


\section{References}

1. Lubricant at the Frontier Tribology, series 36, Editor; D. Dowson, Elsevier Science, B.V., 1999, ISBN:044450267X (Vol.36).

2. Woydt, M., Wasche, R., Wear, 2010, 268, 1542.

3. Lu, J. C.; Thomas, R. K.; Penfold, J., Adv. Coll. Int. Sci., 2000, 84, 143.

4 Penfold, J., Staples, E., Tucker, I., Thompson, L. and Thomas, R.K. J. Coll. Int. Sci., 2002, $247,404$.

5. Bell, G. R.; Bain, C. D.; and Ward, R. N., J. Chem. Soc., Faraday Trans, 1996, 92, 515.

6. Sheasby, J. S. and Caughin, T. A.; Wear, 1991, 150, 247.

7. Armstrong, D. R.; Ferrari, E. S.; Roberts, K. J.; Admas, D., Wear, 1997, 208, 138.

8. Penfold, J and Thomas, R.K., J. Phys.: Condens. Matter, 1990, 2, 1369.

9. Beach, G. S. D.; Parker, F. T.; Smith, D. J.;Crozier, P.A.; Berkowitz, A. E.; Phys Rev Lett, 2003, 91, 267201.

10. Kajdas, C. and Majzner, M., Lubrication Science, 2001, 14, 83. 


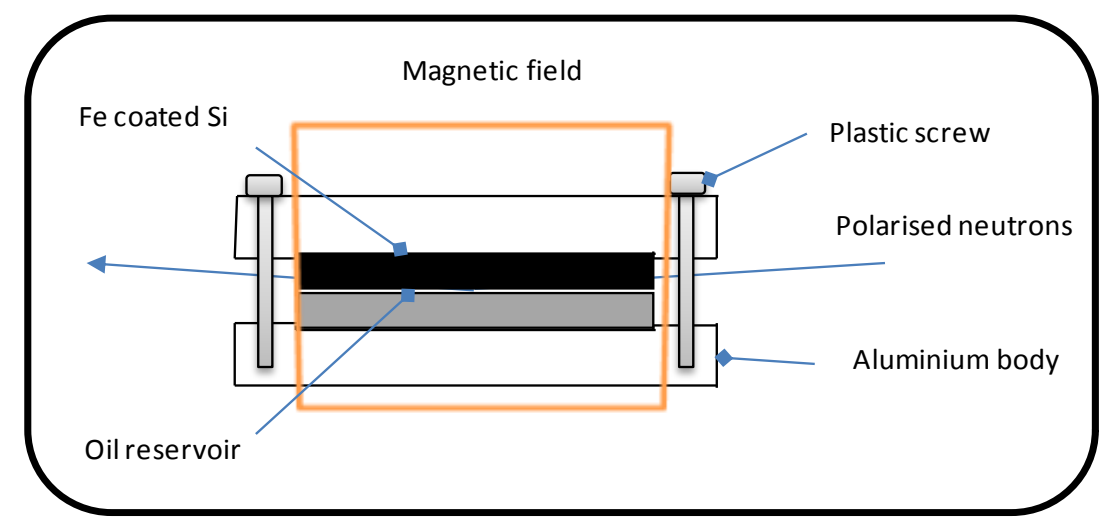

Figure 1. A schematic figure of the reflectivity cell used for the polarised neutron experiment.

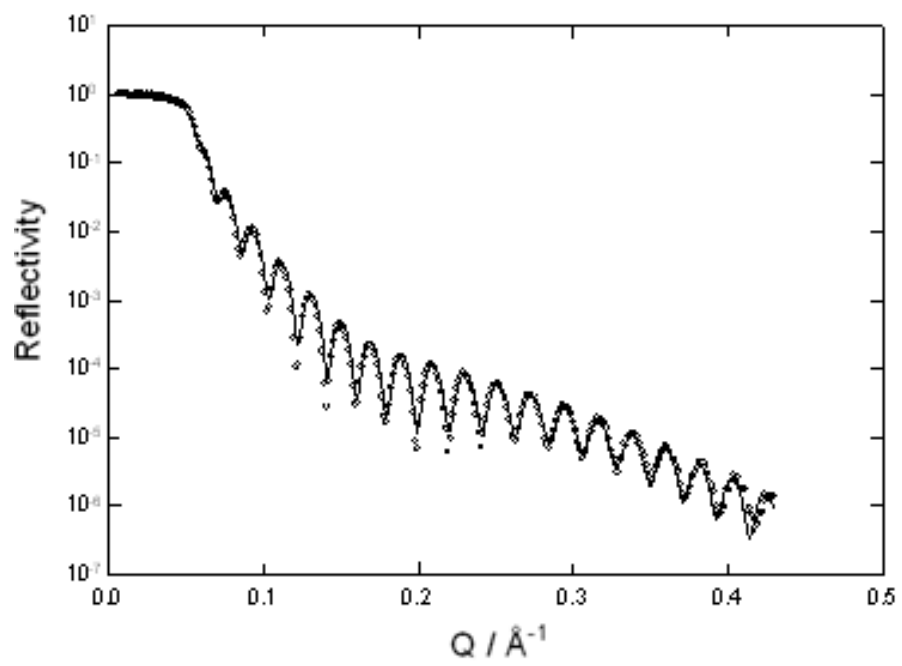

Figure 2. X-ray reflectivity for the calibration sample sputtered on a float glass substrate. The solid line is a fit to the data. The fitted parameters are given in Table 1. 


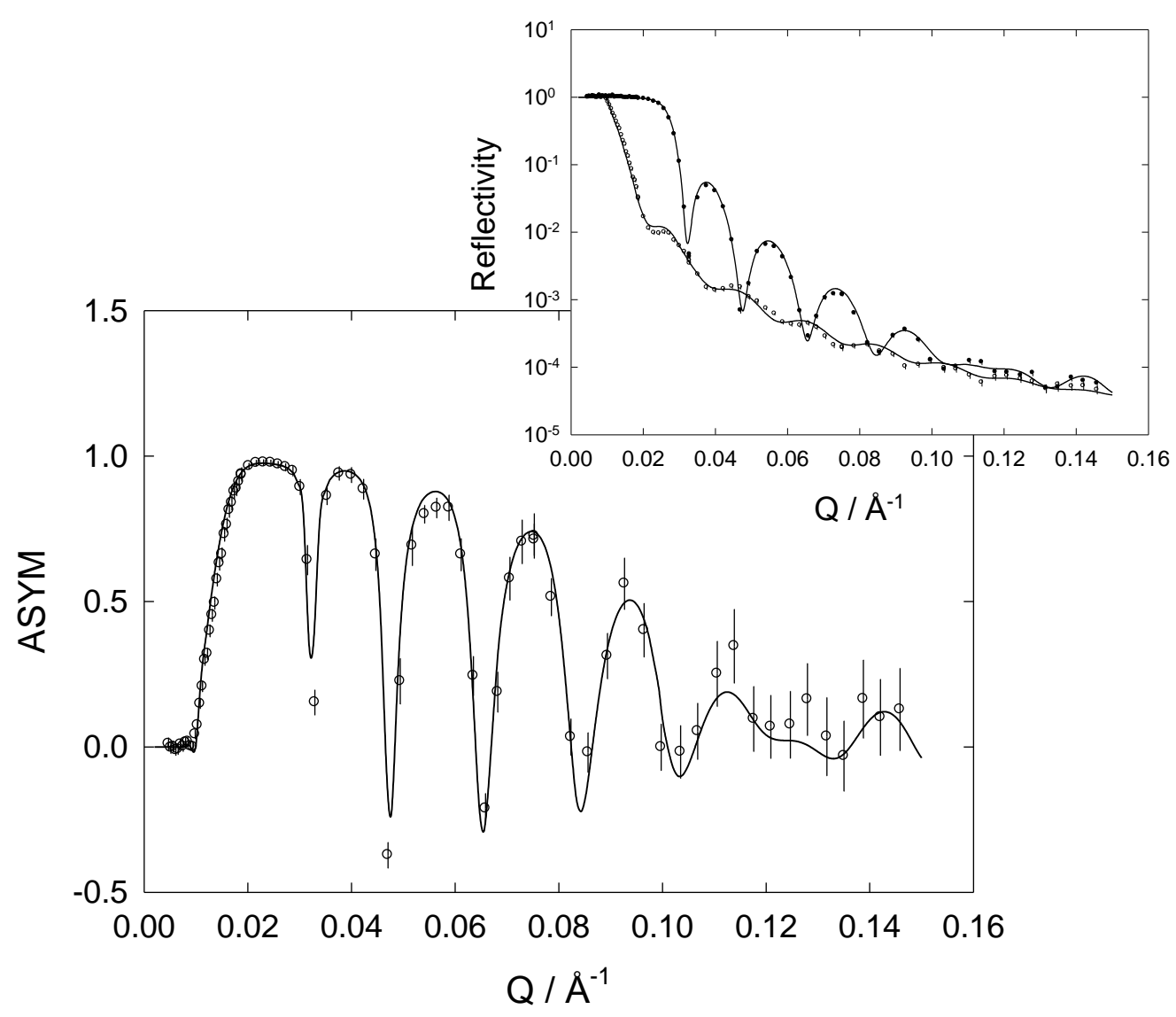

Figure 3. The spin asymmetry (ASYM) $\left(\left(\mathrm{R}^{+}-\mathrm{R}^{-}\right) /\left(\mathrm{R}^{+}+\mathrm{R}^{-}\right)\right)$for the substrate characterisation as a function of the scattering wave-vector $(\mathrm{Q})$ is shown. The open circles are the data and the fit is shown by the solid line. The reflectivity profiles for spin up $\left(\mathrm{R}^{+}\right)(\mathbf{O})$ and spin down $\left(\mathrm{R}^{-}\right)$ (O) neutrons at the air/metal interface are shown as an insert. The solid lines correspond to the simultaneous fit to the data. 
(a)
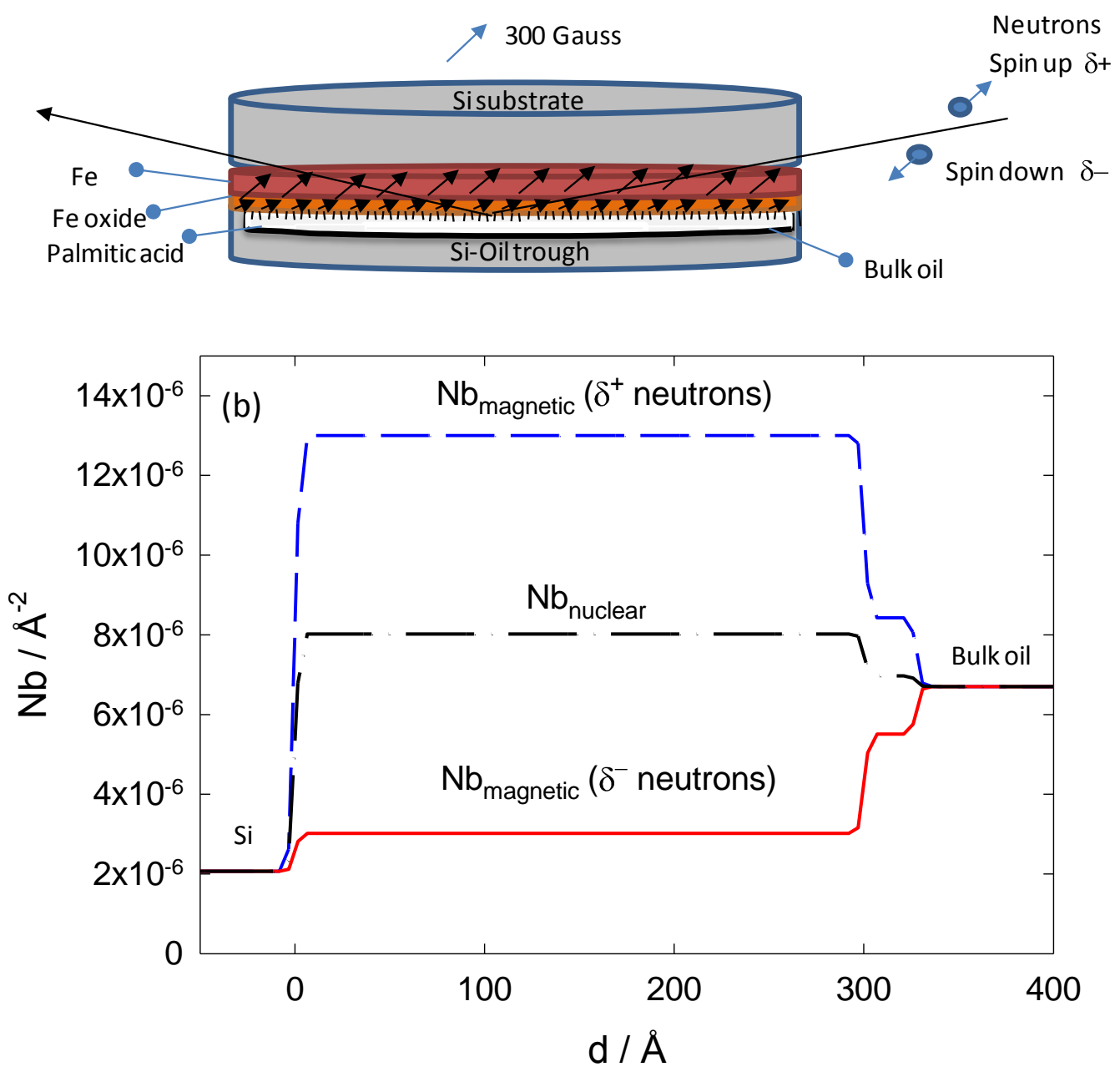

Figure 4. (a) A schematic figure of the experimental geometry used. (b) Idealised neutron scattering length density profiles of the iron coated Si in contact with d-hexadecane. The spin up neutrons (dashed line), spin down neutrons (solid line) and non-polarised neutrons (dashed-dotted line). The contrast using spin down neutrons minimises the reflection from the $\mathrm{Si} /$ iron interface, allowing the adsorption of palmitic acid onto the iron oxide/oil interface to be dominant. 


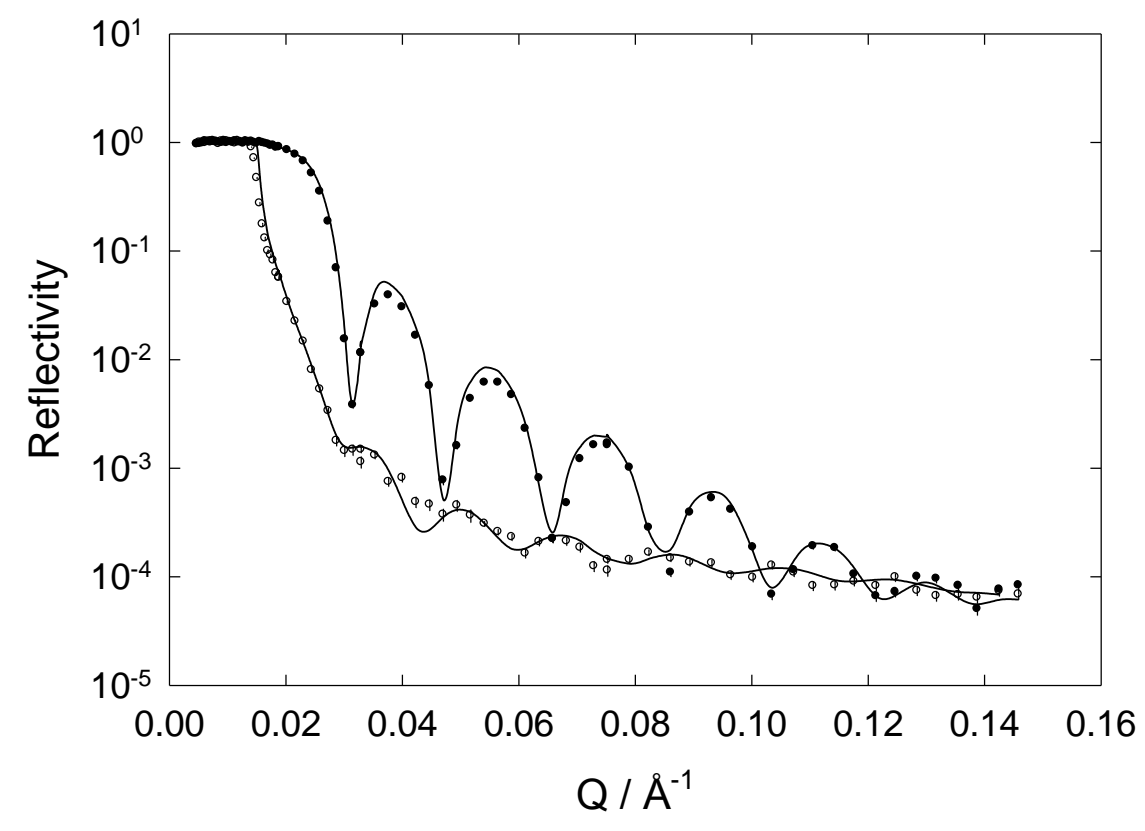

Figure 5. The spin up $(\bigcirc)$ and spin down $(O)$ reflectvities from $150 \mathrm{ppm}$ palmitic acid solution in d-hecadecane against $\mathrm{Si} / \mathrm{Fe} / \mathrm{Fe}_{2} \mathrm{O}_{3}$ interface. 


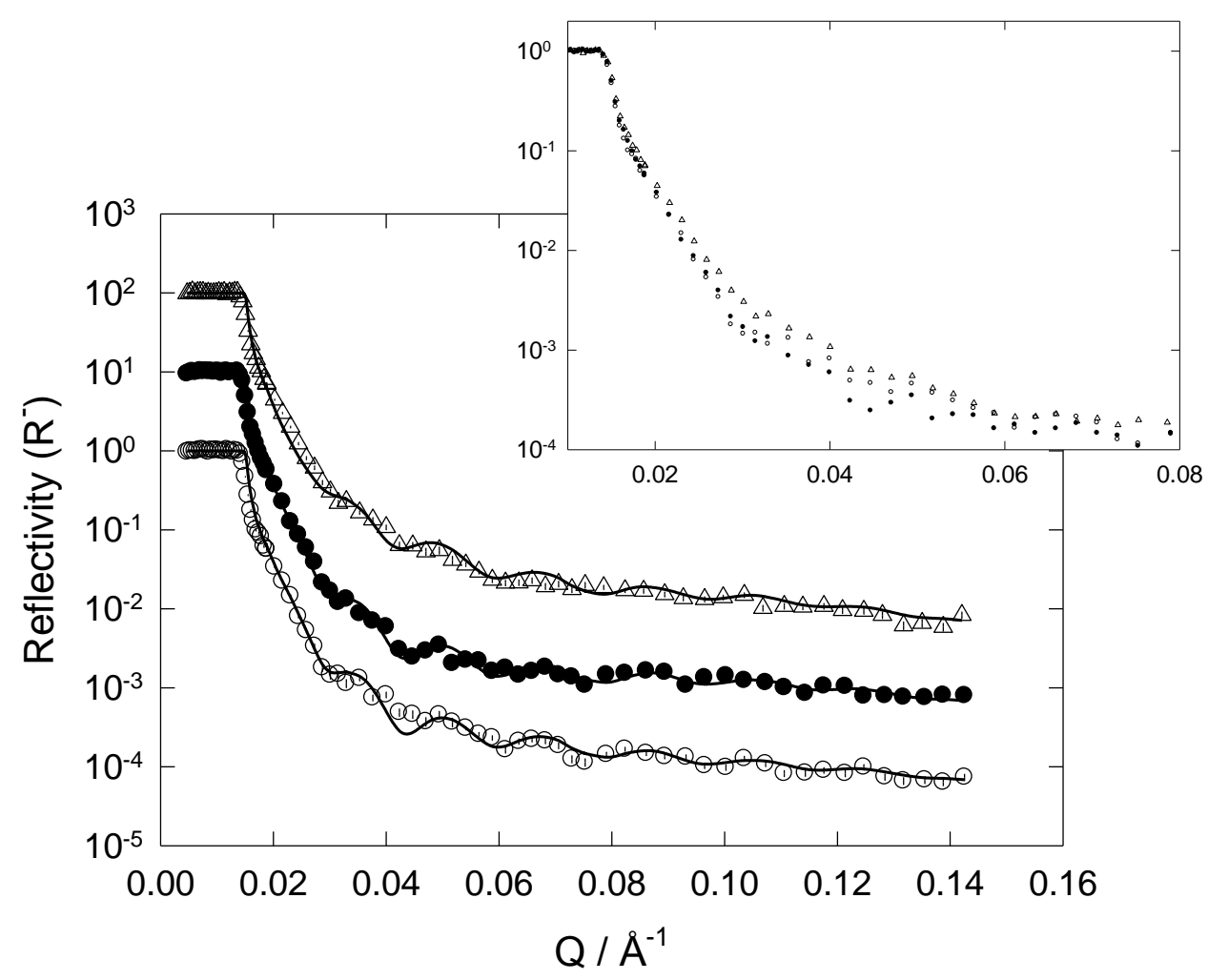

Figure 6. Reflectivity profiles for spin down neutrons for palmitic acid concentrations, $1000 \mathrm{ppm}(\triangle), 500 \mathrm{ppm}\left(\mathrm{O}_{)}\right.$and $150 \mathrm{ppm}(\mathrm{O})$ in d-hexadecane are shown. The fits to the data are shown by the solid lines. The fitted parameters are given in Table 2 . The profiles are shifted by a factor of 10 for the purpose of clarity. The data is also shown (insert) on the same plot to highlight the changes in the reflectivity profiles as a function of concentration. 


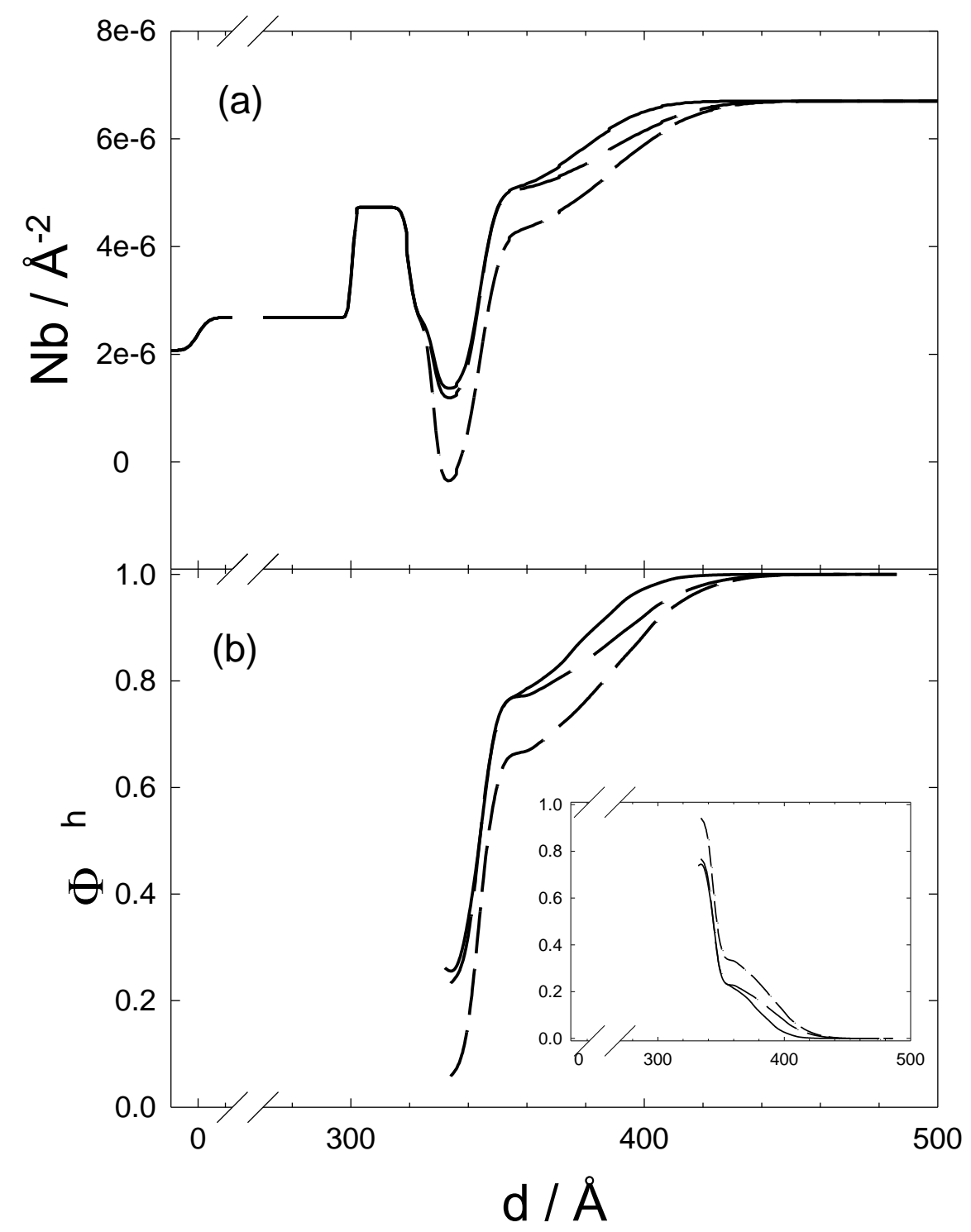

Figure 7(a). Scattering length density profiles for spin down neutrons for the palmitic acid in $\mathrm{d}$-hexadecane. The introduction of a break in the scale is only to highlight the iron oxide/ oil interface. (b) Shows the volume fraction of hexadecane $\left(\Phi_{\mathrm{h}}\right)$ at the interface, whereas the volume fraction for palmitic acid $\left(\Phi_{\mathrm{PA}}=1-\Phi_{\mathrm{h}}\right)$ is shown in the figure inset. Short dash line (150 ppm), dashed line (500ppm), solid lline (1000ppp palmitic acid). 


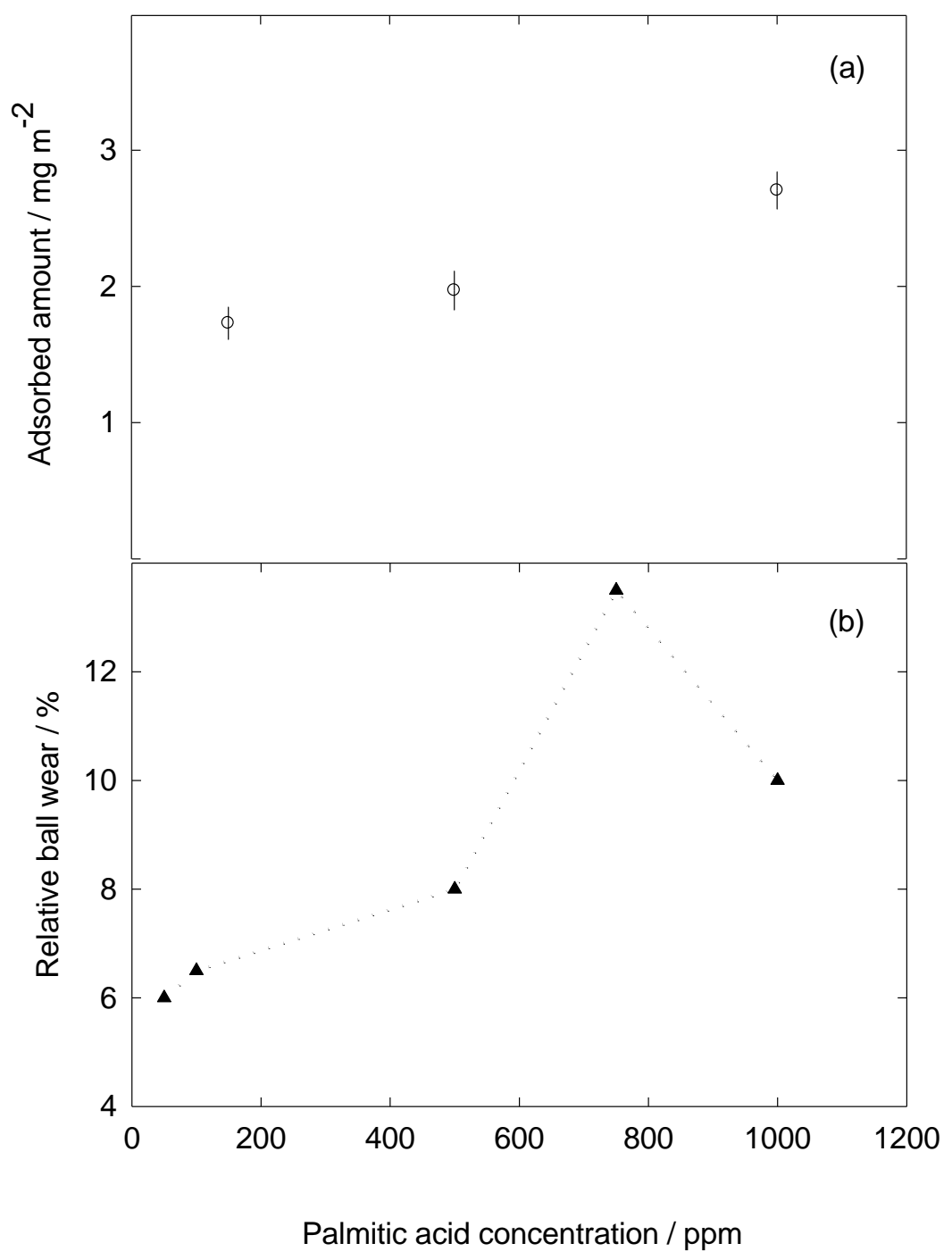

Figure 8. (a) Adsorbed amount as calculated from the scattering length density profiles obtained from the polarised neutron reflectivity. (b) Relative ball wear data published in the literature [10]. 


\begin{tabular}{|c|c|c|c|}
\hline Material & Thickness $/ \AA( \pm 1)$ & Roughness $/ \AA$ & $\mathrm{Nb} \times 10^{-6} / \AA^{-2}$ \\
\hline $\mathrm{Fe}_{2} \mathrm{O}_{3}$ & 21.0 & 5.0 & 3.93 \\
\hline $\mathrm{Fe}$ & 259.0 & 4.0 & 5.94 \\
\hline $\mathrm{Sn}$ & 10.0 & 4.0 & 2.70 \\
\hline Float glass & Bulk & 4.5 & 1.89 \\
\hline
\end{tabular}

Table 1. Structural parameters of the iron coated float glass as determined from X-ray reflectivity

\begin{tabular}{|c|c|c|c|c|}
\hline Layer & Thickness / $\AA( \pm 2)$ & Roughness / & $\begin{array}{c}\text { Magnetic moment } \\
\mu / \mu_{\mathrm{B}}\end{array}$ & $\mathrm{Nb} \times 10^{-6} / \AA^{-2}$ \\
\hline $\mathrm{Air}$ & $/$ & 6 & $/$ & 0 \\
\hline $\mathrm{Fe}_{2} \mathrm{O}_{3}$ & 28 & 1 & 2 & 6.19 \\
\hline $\mathrm{Fe}$ & 300 & 6 & 2.2 & 7.72 \\
\hline $\mathrm{Si}$ & $/$ & $/$ & $/$ & 2.07 \\
\hline
\end{tabular}

Table 2. Structural parameters of the Si/iron/air interface.

\begin{tabular}{|c|c|c|c|c|}
\hline Layer & Thickness / $\AA( \pm 2)$ & $\begin{array}{c}\text { Roughness / } \\
\AA\end{array}$ & $\begin{array}{c}\text { Magnetic moment } \\
\mu / \mu_{\mathrm{B}}\end{array}$ & $\mathrm{Nb} \times 10^{-6} / \AA^{-2}$ \\
\hline \multicolumn{5}{|c|}{ Common layers to all three concentration } \\
\hline $\mathrm{Si}$ & / & 6 & l & 2.07 \\
\hline $\mathrm{Fe}$ & 300 & 1 & 2.2 & 7.72 \\
\hline $\mathrm{Fe}_{2} \mathrm{O}_{3}$ & 20 & 2 & 2 & 6.19 \\
\hline Oxide + PA & 8 & 2 & 1.2 & 3.50 \\
\hline \multicolumn{5}{|c|}{ Palmitic concentration $150 \mathrm{ppm}$} \\
\hline PA & 16 & 5 & l & -0.45 \\
\hline PA + d-hexadecane & 45 & 22 & / & 4.11 \\
\hline d-hexadecane & l & I & l & 6.70 \\
\hline \multicolumn{5}{|c|}{ Palmitic concentration $500 \mathrm{ppm}$} \\
\hline PA & 16 & 4 & l & 1.16 \\
\hline $\mathrm{PA}+\mathrm{d}$-hexadecane & 45 & 22 & I & 4.93 \\
\hline d-hexadecane & I & l & / & 6.70 \\
\hline \multicolumn{5}{|c|}{ Palmitic concentration $1000 \mathrm{ppm}$} \\
\hline PA & 16 & 4 & / & 1.34 \\
\hline PA + d-hexadecane & 35 & 17 & l & 4.93 \\
\hline d-hexadecane & l & l & l & 6.70 \\
\hline
\end{tabular}

Table 3. Structural parameters determined from the model fits to the Si/iron/palmitic acid/deuterated Hexadecane reflectivity profiles. 


\section{TOC}

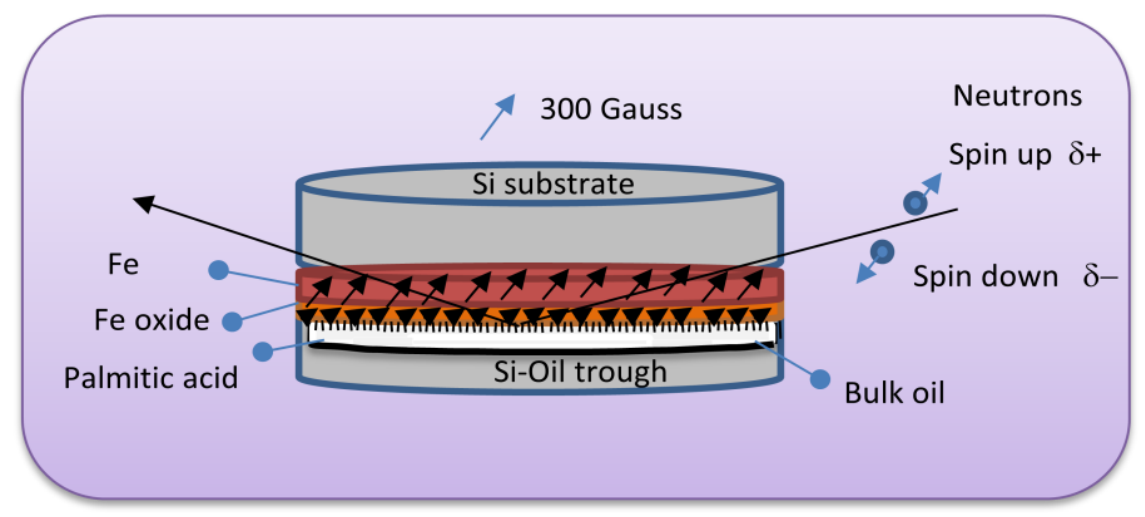

Page 17 of 17 